\title{
Hipoparatireoidismo secundário ao uso prolongado de omeprazol em um cão: relato de caso
}

[Secondary hypoparathyroidism to omeprazole prolonged use in a dog: case report]

\author{
B.C. Aléssio ${ }^{1}$, M.I.P. Palumbo ${ }^{2 *}$, A.I. Souza ${ }^{2}$, P.A.T. Andreussi ${ }^{2}$, \\ P.A. Franco ${ }^{3}$, V.J.B. Terra ${ }^{2}$ \\ ${ }^{1}$ Residente - Universidade Federal de Mato Grosso do Sul - Campo Grande, MS \\ ${ }^{2}$ Universidade Federal de Mato Grosso do Sul - Campo Grande, MS \\ ${ }^{3}$ Autonômo - Campo Grande, MS
}

B.C. Aléssio1,

https://orcid.org/0000-0003-2479-8443 M.I.P. Palumbo2*,

https://orcid.org/0000-0002-0919-5057 A.I. Souza2,

https://orcid.org/0000-0002-8372-6047 P.A.T. Andreussi2, https://orcid.org/0000-0002-9512-4860 P.A. Franco3,

https://orcid.org/0000-0001-9588-9241 V.J.B. Terra2,

https://orcid.org/0000-0002-7794-5163

\section{RESUMO}

O hipoparatireoidismo, quer seja primário ou secundário, é uma doença rara em cães, causada pela diminuição da secreção de paratormônio pelas paratireoides, que leva a sinais clínicos resultantes da hipocalcemia. $\mathrm{O}$ omeprazol vem sendo cada vez mais utilizado na medicina veterinária visando à diminuição na produção de líquor, mas existem poucos estudos sobre os efeitos colaterais relacionados ao uso crônico dessa medicação. Relata-se o caso de um cão macho da raça Yorkshire Terrier, com quatro anos de idade, com sinais clínicos de dor, sendo verificada calcificação em pelve e divertículo renal. Segundo o proprietário, o cão fazia uso de omeprazol há mais de dois anos devido ao histórico de hidrocefalia. Os exames laboratoriais evidenciaram anemia microcítica hipocrômica, hipocalemia, hiperfosfatemia, hipocalcemia, hipomagnesemia e hipercalciúria. A dosagem do paratormônio sérico confirmou o hipoparatireoidismo. Após a suspensão do omeprazol, as alterações encontradas nos exames se normalizaram, confirmando que a causa do hipoparatireoidismo era o uso crônico da medicação.

Palavras-chave: cães, hipocalcemia, hipomagnesemia, hipopotassemia paratormônio

\begin{abstract}
Primary or secondary hypoparathyroidism is a rare disease in dogs caused by the decreased secretion of parathormone from the parathyroid glands, leading to clinical signs of hypocalcemia. Omeprazole has been increasingly used in veterinary medicine in order to reduce the production of cerebrospinal fluid, but there are few reports of side effects related to its chronic use. We report a case of a four-year-old male Yorkshire terrier with clinical signs of pain, calcification in the pelvis and renal diverticulum. According to the owner, the dog had been receiving omeprazole for over 2 years because of the history of hydrocephalus. Hematological exams revealed hypochromic microcytic anemia, hypokalemia, hyperphosphatemia, hypocalcemia, hypomagnesemia besides hypercalciuria. The determination of serum parathyroid hormone concentrations confirmed hypoparathyroidism. After interrupting omeprazole, the altered features on the exams returned to normal values, confirming that the cause of hypoparathyroidism was the chronic use of the drug.
\end{abstract}

Keywords: dogs, hypocalcemia, hypomagnesemia, hypopotassemia, parathormone

\section{INTRODUÇÃO}

Hipoparatireoidismo primário, devido à deficiência relativa ou absoluta do paratormônio, é uma condição rara em cães e pode levar a diversas alterações neurológicas e neuromusculares resultantes de hipocalcemia profunda e de moderada hiperfosfatemia. O paratormônio (PTH) é um hormônio

Recebido em 27 de novembro de 2017

Aceito em 7 de dezembro de 2018

*Autor para correspondência (corresponding author)

E-mail: palumboma11@yahoo.com.br polipeptídico secretado pelas células das glândulas paratireoides em situações de hipocalcemia, o qual regula os níveis séricos de cálcio por meio de ações nos ossos e nos rins. Os níveis de paratormônio podem ser determinados pela dosagem das concentrações séricas de cálcio em combinação com a de paratormônio para o diagnóstico definitivo de hipoparatireoidismo (Ettinger e Feldman, 2010). 
Na medicina humana, o hipoparatireoidismo está associado à hipomagnesemia (Hoorn et al., 2010), hipopotassemia, hipocalcemia e ao uso crônico de inibidores de bomba de prótons (Swaminathan, 2015). Na medicina veterinária, há relatos de hipoparatireoidismo secundário em cinco Yorkshires Terriers e em um Shih-Tzu com perda de proteína entérica (Bush et al., 2001), em um Cocker Spaniel Inglês durante quimioterapia (Horn e Irwin, 2000) e em um Spitz Alemão após laringectomia (Henderson et al., 1991). Há também o relato de hipoparatireoidismo idiopático descrito em cinco gatos (Peterson et al., 1991). No Brasil, até o momento, o único caso relatado foi em um Schnauzer, diagnosticado com a forma primária da doença (Freitas et al., 2014).

O omeprazol é um antissecretolítico com capacidade de inibir em até $95 \%$ a produção de ácido clorídrico, bloqueando, de forma não competitiva, a bomba de prótons. Foi o primeiro inibidor da bomba de prótons (IBP) comercializado, sendo indicado para pacientes com hipergastrinemia e úlceras/erosões gástricas e duodenais (Hoogerwerf e Pasricha, 2006).

A substância também tem efeito na diminuição na produção de líquido cefalorraquidiano (LCR), na redução do acúmulo de LCR no sistema ventricular e na redução de sua turbulência (Driver et al., 2013).

Considerando a ausência de casos semelhantes descritos em cães na literatura veterinária consultada, o objetivo deste relato é descrever a ocorrência de hipoparatireoidismo secundário ao uso prolongado de omeprazol em um cão, além das principais alterações laboratoriais que permitiram o diagnóstico definitivo deste caso.

\section{CASUÍSTICA}

Foi atendido, no Hospital Veterinário da Universidade Federal de Mato Grosso do Sul (UFMS), um cão macho da raça Yorkshire Terrier, com quatro anos de idade, diagnosticado anteriormente com hidrocefalia, que fazia uso de omeprazol na dose de $2,9 \mathrm{mg} / \mathrm{kg}$ a cada $24 \mathrm{~h}$, por via oral, há aproximadamente dois anos ininterruptos. Esporadicamente, quando o cão manifestava sinais neurológicos, como andar compulsivo, era medicado com prednisolona, na dose de $1,47 \mathrm{mg} / \mathrm{kg}$, a cada 48 horas, por via oral, durante sete a 10 dias, com remissão dos sinais.
Desta vez, o proprietário buscou atendimento veterinário, pois o animal apresentava crises de dor cuja origem ele não conseguia definir. Foi relatado que o cão ficava inquieto e se contraía quando manipulado, além de apresentar poliúria, polidipsia e abdome abaulado. No exame físico, o animal manifestou sensibilidade à palpação da região lombossacral.

Foram realizados exames laboratoriais que evidenciaram anemia microcítica hipocrômica (Hb 9,2g/dL, ref. 12 a 18g/dL; VG 31,3\%, ref. 37 a $55 \%$; VCM 50,4fL, ref. 60 a $77 \mathrm{fL}$; $\mathrm{CHCM}$ $29,7 \mathrm{~g} / \mathrm{dL}$, ref. 32 a $36 \mathrm{~g} / \mathrm{dL})$. Devido à suspeita de deficiência de ferro, a dosagem de ferro foi solicitada, e o resultado apresentou-se dentro do valor de normalidade $(188 \mathrm{mcg} / \mathrm{dL}$, ref. 84 a $233 \mathrm{mcg} / \mathrm{dL})$. Também se constatou hiperfosfatemia $(6,68 \mathrm{mg} / \mathrm{dL}$, ref. $2,6-6,2 \mathrm{mg} / \mathrm{dL})$, hipocalemia $(3,07 \mathrm{mmol} / \mathrm{L}$, ref. 3,9-5,2 mmol/L), hipocalcemia $(0,195 \mathrm{mmol} / \mathrm{L}, \quad$ ref. 1,15 $1,39 \mathrm{mmol} / \mathrm{L})$ e hipomagnesemia $(1,1 \mathrm{mg} / \mathrm{dL}$, ref. 1,8-2,4mg/dL). A única alteração observada na urinálise foi elevação da razão proteínacreatinina urinária (razão UP/C 0,4 , ref.<0,2). Exames de imagem, como ultrassonografia e radiografia abdominais, indicaram nefropatia parenquimatosa difusa discreta a moderada bilateral, com acentuada mineralização de recessos pélvicos (Fig. 1).

Foi realizado o teste de excreção fracionada de cálcio na urina, que demonstrou resultado compatível com hipercalciúria $(2,21 \%$, ref. 0,66$1,76 \%)$. A dosagem sérica de vitamina D3 foi de 49,7ng/mL (deficiente<24,9ng/mL; limítrofe 25-99,9ng/mL; desejável 100-120ng/mL; aumentado $>120 \mathrm{ng} / \mathrm{mL}$ ) e o nível de paratormônio foi inferior a $1,2 \mathrm{pg} / \mathrm{mL}$ (ref. 19 a 123,8pg/mL), confirmando o diagnóstico de hipoparatireoidismo.

Para o controle da dor, foram prescritos cloridrato de tramadol $(2 \mathrm{mg} / \mathrm{kg}, \mathrm{BID}, \mathrm{VO}) \mathrm{e}$ dipirona $(25 \mathrm{mg} / \mathrm{kg}$, BID, VO), além de encaminhamento para acupuntura. $\mathrm{O}$ proprietário foi orientado a interromper o uso de prednisolona e omeprazol devido à suspeita de as alterações observadas serem secundárias ao uso desses medicamentos. Após a segunda sessão de acupuntura, os analgésicos foram suspensos e o animal não apresentou mais sinais de dor. A poliúria e a polidipsia desapareceram após a interrupção do uso da prednisolona. 


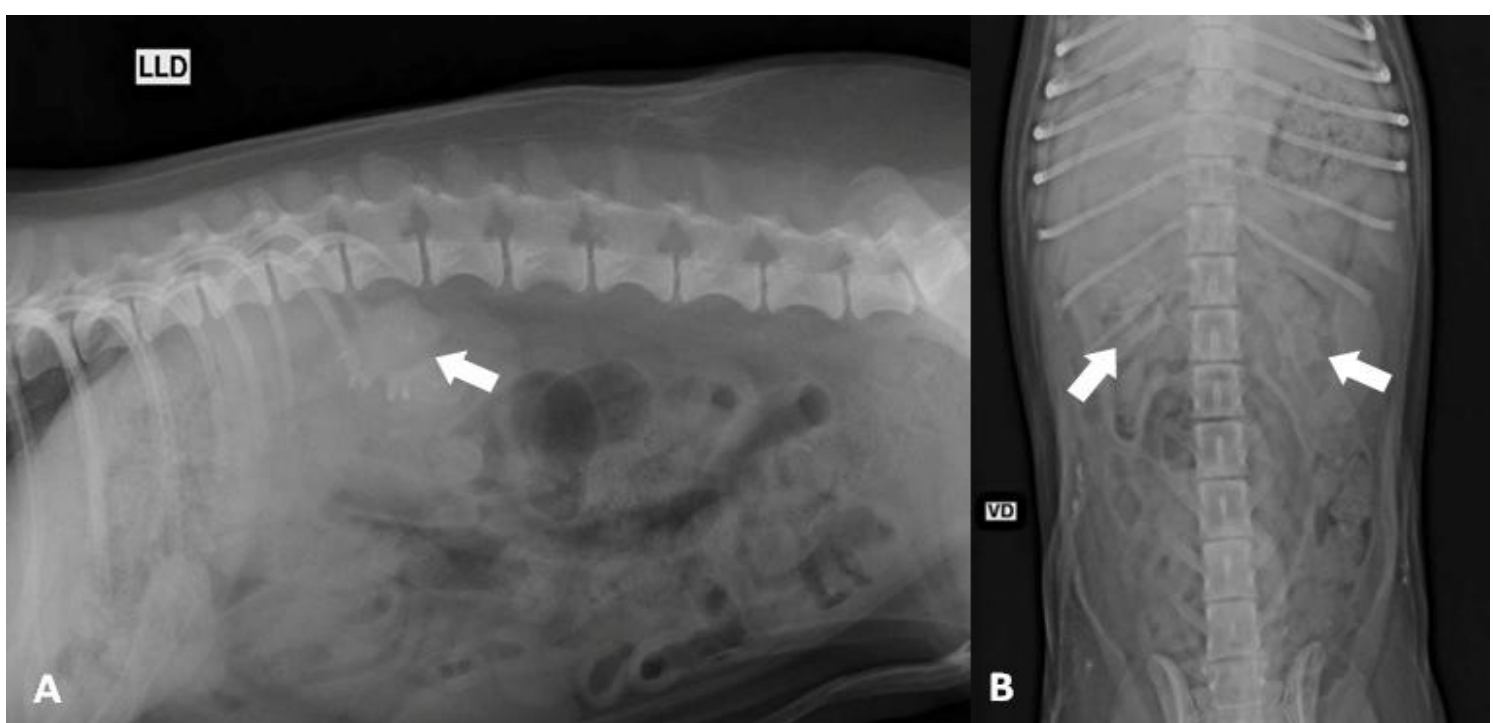

Figura 1. Radiografia abdominal. A) Projeção laterolateral direita. B) Projeção ventrodorsal. Notar a presença de acentuada mineralização bilateral de pelve e divertículos renais (setas).

Após 45 dias sem o uso de omeprazol e prednisolona, o paciente estava assintomático e houve normalização das dosagens séricas de fósforo $(3,6 \mathrm{mg} / \mathrm{dL}$, ref. 2,6 a $6,2 \mathrm{mg} / \mathrm{dL})$, magnésio $(1,8 \mathrm{mg} / \mathrm{dL}$, ref. 1,8 a $2,4 \mathrm{mg} / \mathrm{dL})$, paratormônio $(6,6 \mathrm{pg} / \mathrm{ml}$, ref. 5 a $25 \mathrm{pg} / \mathrm{mL})$ e da taxa de excreção urinária de cálcio $(0,0017 \%$, ref. 0,66 a $1,76 \%$ ), porém ainda havia anemia microcítica hipocrômica $(\mathrm{Hb} 11 \mathrm{~g} / \mathrm{dL}$, ref. 12 a $18 \mathrm{~g} / \mathrm{dL}$; VG $34,5 \%$, ref. 37 a $55 \%$; VCM $52 \mathrm{fL}$, ref. 60 a $77 \mathrm{fL}$; $\mathrm{CHCM} 31,9 \mathrm{~g} / \mathrm{dL}$, ref. 32 a $36 \mathrm{~g} / \mathrm{dL})$, hipocalemia $(3,18 \mathrm{mmol} / \mathrm{L}$, ref. 3,9 a $5,2 \mathrm{mmol} / \mathrm{L})$ e hipocalcemia $(0,404 \mathrm{mmol} / \mathrm{L}$, ref. 1,15 a 1,39mmol/L). Após 65 dias sem omeprazol, o animal não apresentava mais anemia (Hb 12g/dL, ref. 12 a 18g/dL; VG 39,3\%, ref. 37 a 55\%), e houve normalização dos níveis de potássio $(4,15 \mathrm{mmol} / \mathrm{L}$, ref. 3,9 a $5,2 \mathrm{mmol} / \mathrm{L})$, porém o cálcio continuou abaixo do valor de referência $(0,655 \mathrm{mmol} / \mathrm{L}$, ref. 1,15 a $1,39 \mathrm{mmol} / \mathrm{L})$. Nessa ocasião, foi feita a suplementação com calcitriol $(0,04 \mu \mathrm{g} / \mathrm{kg}$, SID, VO) durante sete dias e houve a normalização dos valores de cálcio ionizado $(1,271 \mathrm{mmol} / \mathrm{L}$, ref. 1,15 a $1,39 \mathrm{mmol} / \mathrm{L})$. A Tab. 1 mostra a evolução dos resultados de exames laboratoriais a cada retorno do animal.

Tabela 1. Valores de exames realizados no primeiro dia de atendimento (dia 1), após a interrupção do uso do omeprazol (dia 45 e dia 65) e após suplementação com vitamina D (dia 72)

\begin{tabular}{|c|c|c|c|c|c|}
\hline Parâmetro & Dia 0 & Dia 45 & Dia 65 & Dia 72 & Valores de referência \\
\hline $\mathrm{Hb}(\mathrm{g} / \mathrm{dL})$ & 9,2 & 11 & 12 & - & 12 a $18 \mathrm{~g} / \mathrm{Dl}$ \\
\hline VG (\%) & 31,3 & 34,5 & 39,3 & - & 37 a $55 \%$ \\
\hline VCM (fL) & 50,4 & 52 & - & - & 60 a $77 \mathrm{Fl}$ \\
\hline CHCM & 29,4 & 31,9 & - & - & 32 a $36 \mathrm{~g} / \mathrm{Dl}$ \\
\hline Ferro sérico & 188 & - & - & - & 88 a $233 \mathrm{mcg} / \mathrm{dL}$ \\
\hline Razão UP/C & 0,46 & - & - & - & $<0,2$ \\
\hline Fósforo & 6,68 & 3,6 & - & - & 2,6 a $6,2 \mathrm{mg} / \mathrm{Dl}$ \\
\hline Potássio $\left(\mathrm{K}^{+}\right)$ & 3,07 & 3,18 & 4,15 & - & 3,9 a $5,2 \mathrm{mmol} / \mathrm{L}$ \\
\hline Cálcio $\left(\mathrm{Ca}^{2+}\right)$ & 0,195 & 0,404 & 0,655 & 1,271 & 1,15 a $1,39 \mathrm{mmol} / \mathrm{L}$ \\
\hline Magnésio (Mg) & 1,1 & 1,8 & - & - & 1,8 a $2,4 \mathrm{mg} / \mathrm{Dl}$ \\
\hline PTH & $<1,2$ & 6,6 & - & & 5 a $25 \mathrm{pg} / \mathrm{Ml}$ \\
\hline $\begin{array}{l}\text { Excreção frac. Cálcio } \\
\text { na urina }\end{array}$ & $2,21 \%$ & 0,0017 & - & & 0,66 a $1,76 \%$ \\
\hline Vitamina D3 & - & 49,7 & - & - & 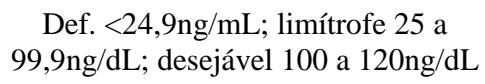 \\
\hline Vitamina B12 & - & 179 & - & - & 175 a $550 \mathrm{pg} / \mathrm{mL}$ \\
\hline
\end{tabular}




\section{DISCUSSÃO}

A ocorrência de hipoparatireoidismo secundário ao uso crônico de omeprazol já foi descrita em seres humanos (Epstein et al., 2006; Swaminathan, 2015). Na medicina veterinária, o omeprazol oral tem sido recomendado para reduzir a produção do LCR em cães acometidos por desordens cujos sinais clínicos podem ser atribuíveis ao acúmulo de LCR no sistema nervoso central, como na hidrocefalia (Driver et al., 2013). O animal deste estudo recebia omeprazol por período prolongado como terapia conservadora de hidrocefalia, o que possivelmente resultou no desenvolvimento de hipoparatireoidismo secundário ao uso crônico de omeprazol, caso ainda não descrito na literatura consultada.

O diagnóstico definitivo de hipoparatireoidismo neste caso foi confirmado com base no achado de níveis séricos reduzidos de paratormônio que, segundo Ettinger e Feldman (2010), são dados confirmatórios, embora Nelson e Couto (2009) digam que se deve suspeitar da doença em cães ou gatos que apresentem hipocalcalcemia persistente, hiperfosfatemia e função renal normal. Após a suspensão do uso do omeprazol, houve a normalização dos níveis séricos do paratormônio, bem como dos eletrólitos $\left(\mathrm{Ca}^{2+}\right.$, $\mathrm{Mg}^{2+}, \mathrm{K}^{+}$), o que permite inferir que as alterações eram secundárias a esse medicamento, já que nenhum tratamento específico foi estabelecido.

A dor abdominal apresentada pelo paciente deste relato já foi relatada por Russell e colaboradores (2006) em cães com hipoparatireoidismo. Outros sinais clínicos também podem estar presentes, como convulsões, vômito, ataxia, alteração de comportamento e hipertermia, mas não foram observados neste caso. As outras alterações relatadas, incluindo poliúria, polidipsia e aumento de volume abdominal, possivelmente estão relacionadas à administração excessiva de glicocorticoides, conforme descrito por Ettinger e Feldman (2010).

A anemia microcítica hipocrômica apresentada pelo animal foi resolvida sem intervenção terapêutica, além da suspensão do omeprazol, porém na literatura consultada não há relatos sobre essa relação. Tempel et al. (2013) demonstraram que o uso de omeprazol em curto período de tempo não causou alteração nos níveis séricos de ferro, porém Golubov et al. (1991) concluíram que essa medicação não causa alteração na absorção de ferro em pacientes saudáveis, mas reduz sua absorção em indivíduos doentes, levando à deficiência desse nutriente. Possivelmente, este é o motivo pelo qual o animal em questão apresentava o ferro sérico dentro do valor de normalidade, por ser um animal sem outras afecções concomitantes.

A deficiência do paratormônio diminui a reabsorção tubular de cálcio pelos rins (Nelson e Couto, 2009), o que justifica a excreção fracionada de cálcio na urina que estava elevada no primeiro dia de consulta, mas que se normalizou 45 dias após suspensão do omeprazol. A mineralização da pelve e dos divertículos renais registrada pela radiografia pode ser a origem da dor abdominal que o animal apresentava e, possivelmente, deve-se à hipercalciúria. $\mathrm{O}$ paratormônio também exerce papel na transformação da forma inativa para a forma ativa da vitamina $\mathrm{D}$ (1,25 di-hidroxivitamina D3), que, por sua vez, tem como função intensificar a absorção intestinal de cálcio (Moreira et al., 2004). O cão deste caso apresentou valores limítrofes da vitamina $\mathrm{D}$, fato que provavelmente colaborou para o desenvolvimento de hipocalcemia.

A literatura acerca das alterações hematológicas e eletrolíticas observadas em animais que são medicados com omeprazol é escassa. Apesar de o estudo realizado por Gould et al. (2016) em gatos ter concluído que, após 60 dias de uso de omeprazol, não ocorreu diminuição dos níveis séricos de magnésio, cálcio e vitamina B12, pouco se sabe sobre as alterações observadas com utilização desse medicamento durante dois anos em cães, que foi o período fornecido para o animal deste relato. Nos casos humanos, a ocorrência de hipoparatireoidismo foi observada após dois dias de sua administração intravenosa (Swaminathan, 2015). Quando seu uso foi pela via oral, as alterações deram-se após dois anos (Epstein et al., 2006) de uso de IBPs. Como no presente caso só foram realizados exames laboratoriais dois anos após o uso do omeprazol, não é possível determinar em quanto tempo as alterações começaram a ocorrer.

Desde 2006, há um crescente aumento de trabalhos evidenciando que os IBPs induzem a hipomagnesemia em pacientes humanos que 
fazem seu uso crônico indiscriminadamente, pela diminuição da absorção intestinal de $\mathrm{Mg}^{2+}$ (Epstein et al., 2006; Shabajee et al., 2008; Cundy e Mackay, 2011). A suplementação oral de $\mathrm{Mg}^{2+}$ não resultou em aumento dos seus níveis no plasma, sendo necessária sua administração intravenosa (Epstein et al., 2006; Shabajee et al., 2008; Cundy e Mackay, 2011). Entretanto, nesses casos, a hipomagnesemia foi rapidamente resolvida quando o uso do omeprazol foi descontinuado (Epstein et al., 2006; Cundy e Mackay, 2011), fato também observado no paciente deste relato.

Hoorn e colaboradores (2010) relataram quatro casos em humanos que recebiam IBP por mais de um ano, nos quais houve indução de hipomagnesemia e hipocalemia por perda de potássio renal. Distúrbios eletrolíticos como hipocalcemia, hipocalemia e hiperfosfatemia, além de hipoparatireoidismo funcional, estão relacionados à hipomagnesemia, segundo Shabajee et al. (2008). Em casos de hipomagnesemia, para diferenciar se há perda renal ou gastrointestinal, o magnésio urinário também deve ser determinado (Cundy e Mackay, 2011). Entretanto, no presente caso, não foi possível realizar a dosagem de magnésio urinário para se determinar o mecanismo da hipomagnesemia.

A hipocalemia está presente em 40-60\% de indivíduos com hipomagnesemia. O magnésio inibe a excreção luminal de potássio, portanto sua deficiência leva ao aumento da eliminação do potássio, sendo sua suplementação refratária se não realizada correção dos níveis de magnésio (Whang et al., 1992). Neste caso, 45 dias após a suspensão do omeprazol, o nível sérico de potássio ainda estava abaixo do esperado e normalizou-se nos exames realizados no dia 65 .

\section{CONCLUSÃO}

Este é o primeiro relato de hipoparatireoidismo induzido pelo uso crônico de omeprazol em um cão. As principais alterações laboratoriais observadas foram: anemia microcítica hipocrômica, hipocalemia, hiperfosfatemia, hipocalcemia, hipomagnesemia, hipercalciúria e nível sérico baixo de paratormônio. $\mathrm{O}$ caso descrito reforça a necessidade de cautela na prescrição de inibidores de bomba de prótons por períodos prolongados em cães, sob risco de desenvolvimento de hipoparatireoidismo.

\section{REFERÊNCIAS}

BUSH, W.W; KIMMEL, S.E.; WOSAR, M.A.; JACKSON, M.W. Secondary hypoparathyroidism attributed to hypomagnesemia in a dog with protein-losing enteropathy. J. Am. Vet. Med. Assoc., v.219, p.1732-1734, 2001.

CUNDY, T.; MACKAY, J. Proton pump inhibitors and severe hypomagnesemia. Curr. Opin. Gastroenterol., v.27, p.180-185, 2011.

DRIVER, C.J.; VOLK, H.A.; RUSBRIDGE, C.; VAN HAM, L.M. An update on the pathogenesis of syringomyelia secondary to Chiari-like malformations in dogs. Vet. J. v.198, p.551-559, 2013.

EPSTEIN, M.; MCGRATH, S.; LAW, F. Proton pump inhibitors and hypomagnesemia hypoparathyroidism. N. Engl. J. Med., v.355, p.1834-1836, 2006.

ETTINGER, S.J.; FELDMAN, E.C. Disorders of the parathyroid glands. In: FELDMAN, E.C. Textbook of veterinary internal medicine diseases of the dog and the cat. 7.ed. St Louis: Saunders Elsevier, 2010. v.2, p.1382-1394.

FREITAS, M.A.; JARK, P.C.; PALUMBO, M.I.P. et al. Hipoparatireoidismo primário em cão. Semin. Ciênc. Agrár., v.35, p.387-392, 2014.

GOLUBOV, J.; FLANAGAN, P.; ADAMS, P. Inhibition of iron absorption by omeprazole in rat model. Dig. Dis. Sci., v.36, p.405-408, 1991.

GOULD, E.; CLEMENTS, C.; REED, A. et al. A prospective, placebo-controlled pilot evaluation of the effect of omeprazole on serum calcium, magnesium, cobalamin, gastrin concentrations, and bone in cats. J. Vet. Intern. Med., v.30, p.779-786, 2016.

HENDERSON, R.A.; POWERS, R.D.; PERRY, L. Development of hypoparathyroidism after excision of laryngeal rhabdomyosarcoma in a dog. J. Am. Vet. Med. Assoc., v.198, p.639-643, 1991. 
HOOGERWERF, W.A.; PASRICHA, P.J. In: GOODMAN, A.G. (Ed.). The pharmacological basis of therapeutics. 11.ed. New York: Mc Graw Hill, 2006. p.967-981.

HOORN, E.J.; HOEK, J.V.D.; MAN, R.A. et al. A case series of proton pump inhibitor-induced hypomagnesemia. Am. J. Kidney Dis., v.56, p.112-116, 2010.

HORN, B.; IRWIN, P.J. Transient hypoparathyroidism following successful treatment

MOREIRA, R.O.; DUARTE, M.P.C.; FARIAS, M.L.F. Distúrbios do eixo cálcio - PTH vitamina D nas doenças hepáticas crônicas. Arq. Bras. Endocrinol. Metabol., v.48, p.443-450, 2004.

NELSON, R.W.; COUTO, C.G. Disorders of the parathyroid gland. In: Small animal internal medicine. 4.ed. Loius, Missouri: Mosby Elsevier, 2009. chap.50, p.715-723, 2009.

of hypercalcaemia of malignancy in a dog. Aust. Vet. J., v.78, p.690-692, 2000.
PETERSON, M.E.; JAMES, K.M.; WALLACE, M. et al. Idiopathic hypoparathyroidism in five cats. J. Vet. Intern. Med., v.5, p.47-51, 1991.

RUSSELL, N.J.; BOND, K.A.; ROBERTSON, I.D. et al. Primary hypoparathyroidism in dogs: a retrospective study of 17 cases. Aust. Vet. J., v.84, p.285-290, 2006.

SHABAJEE, N.; LAMB, E.J.; STURGESS, I.; SUMATHIPALA, R.W. Omeprazole and refractory hypomagnesemia. BMJ. v.10, p.337425, 2008.

SWAMINATHAN, K. Proton pump inhibitorinduced hypomagnesemic hypoparathyroidism. Indian J. Pharmacol., v.47, p.330-331, 2015.

TEMPEL, M.; CHAWLA, A.; MESSINA, C.; ÇELIKER, M.Y. Effects os omeprazole on iron absorption: preliminar study. Turk. J. Hematol., v.30, p.307-310, 2013.

WHANG, R.; WHANG, D.D.; RYAN, M.P. Refractory potassium repletion. A consequence of magnesium deficiency. Arch. Intern. Med., v.152, p.40-45, 1992. 